\title{
Renoprotective Effect of Co-Enzyme Q10 and N-Acetylcysteine on Streptozotocin-Induced Diabetic Nephropathy in Rats
}

\author{
Manojkumar S Mahajan ${ }^{1 *}$, Chandrashekhar D Upasani', Aman B Upaganlawar ${ }^{1}$ and Visha
${ }^{1}$ Department of Pharmacology, SNJB's Shriman Sureshdada Jain College of Pharmacy, Savitribai Phule
Pune University, India
}

${ }^{2}$ School of Pharmaceutical Sciences, Sandip University, India

*Corresponding author: Manojkumar S Mahajan, Assistant Professor, SNJB's Shriman Sureshdada Jain College of Pharmacy, Savitribai Phule Pune University, India, Tel: +91-9423962663

\begin{abstract}
Background: Persistent chronic hyperglycemia is an important player in the development and progression of diabetic nephropathy (DN) due to generation of oxidative stress (OS) which is at the center in the pathophysiology of DN and underlying kidney damage. The present study was aimed to evaluate the effect of antioxidants like Co-enzyme Q10 (CoQ10) and N-acetylcysteine (NAC) either alone or in combination in streptozotocin (STZ) induced diabetic nephropathy $(\mathrm{DN})$ in rats.

Methods: Type-1 diabetes mellitus (T1DM) was induced in male Sprague-Dawley rats by intraperitoneal (i.p.) administration of $55 \mathrm{mg} / \mathrm{kg} \mathrm{STZ}$. DN was confirmed by assessment of renal function tests. Blood glucose level, glycated hemoglobin $(\mathrm{HbA} 1 \mathrm{c})$, serum and urinary total protein, albumin, creatinine, urea, blood urea nitrogen (BUN) and uric acid were determined. Markers of oxidative stress including superoxide dismutase (SOD), catalase (CAT), malondialdehyde (MDA), glutathione (GSH), myeloperoxidase (MPO) and nitrite content in renal homogenate were measured. Histopathological evaluation of kidney was carried to assess renal damage. The rats were treated with CoQ10 (10 mg/ $\mathrm{kg}$, p.o.) alone or in combination with NAC (300 mg/kg, p.o.) for 8 weeks after confirmation of DN.

Results: Renal function of diabetic rats was significantly impaired as indicated by renal function tests than control rats. Renal damage caused due to STZ was identified by means of increased MDA, depleted SOD and CAT activities and reduced GSH. MPO activity and nitrite content in rats with DN increased significantly. Treatment with CoQ10 or NAC and their combined treatment improved STZ induced renal damage as reflected by reduced oxidative stress. Also, the combined treatment protected renal structural damage as seen in histopathological assessment.
\end{abstract}

Conclusion: The present research suggests that oxidative stress due to persistent hyperglycemia causes development and progression of DN whereas combined administration of antioxidants used in the current investigation i.e. CoQ10 and NAC has better renoprotective effect by attenuation of DN than coenzyme Q10 or NAC alone.

\section{Keywords}

Diabetic nephropathy, Streptozotocin, Coenzyme Q10, $\mathrm{N}$-acetylcysteine, Oxidative stress, Renal function

\section{Introduction}

Diabetes mellitus (DM) is a metabolic disorders characterized chronic hyperglycemia due to either reduced glucose import and uptake resulting from decrease in circulating levels of insulin i.e. insulin deficiency or decreased tissue sensitivity to it leading insulin resistance or combination of both deficiency and resistance. DM is categorized as type 1 (T1DM) and type 2 DM (T2DM) [1]. Both the forms of DM possess a strong genetic component in addition to the acquired pathogenic causes.

Type 1 DM which occurs due to autoimmune destruction of pancreatic $\beta$-cells or absolute deficiency of insulin is also known as insulin dependent diabetes mellitus (IDDM). There is considerable evidence indicating that the chronic hyperglycemia causes many of the chronic complications of DM. There are several macrovascular complications like coronary artery, cerebrovascular and peripheral vascular disease arising due to damaged blood vessels. The primary microvascular ma- 
nifestations of DM include diabetic nephropathy (DN), diabetic neuropathy and diabetic retinopathy $[2,3]$.

DN is a leading reason for chronic kidney disease (CKD) and end-stage renal disease (ESRD). It is traditionally defined as a glomerular disease inclusive of five distinct stages: Glomerular hyperfiltration, incipient nephropathy, microalbuminuria, overt proteinuria and end-stage renal disease [4]. It is a progressive and irreversible loss of renal function characterized by initial hyperfiltration, albuminuria, glomerular mesangium expansion, accumulation of extracellular matrix, interstitial fibrosis, thickening of basement membranes and renal cell damage. This means, DN leads to structural changes and functional abnormalities in the kidneys [5].

Hyperglycemia is the key player in the development of DN. Recent clinical studies have shown that persistent hyperglycemia in DM can induce oxidative stress through diverse mechanisms including glucose auto-oxidation, protein glycation through non-enzymatic means, polyol pathway activation and acceleration, and reduced antioxidant defense system. Hyperglycemia may act through PKC activation, acceleration of the polyol pathway, production of reactive oxygen species (ROS) and over-expression of transforming growth factor- $\beta$ (TGF- $\beta$ ) [6].

The normal kidney due to its high metabolic activity is capable of generating a considerable oxidative stress that is balanced by an extensive antioxidant system. Accumulating research showed that the significant contributor to the diabetic complications is chronic hyperglycemia that shifts this balance to a pro-oxidant state leading to tissue damage and vascular injury [7]. It is shown that almost all pathways contributing to the DN induce oxidative stress by one or other mechanism.

Presently, the therapies used in the treatment of DN have not been fully efficacious due the diverse etiologies in the development of DN. This results in difficulty in selecting best possible treatment strategy and therapeutic agent. Therefore effective and new therapeutic approaches than available are needed in the treatment of DN. Many studies reported that therapies with antioxidant have a beneficial effect on DN [8].

Coenzyme Q10 (ubiquinone, CoQ10), is an endogenous fat-soluble vitamin like substance serving as natural antioxidant. It acts as a principal player of the electron transport chain (ETC) of the mitochondria responsible for synthesis of ATP [9]. Apart from the proved pharmacological actions of CoQ10 like its antihypertensive action, it is a powerful antioxidant with the potential to scavenge free radical thereby protecting the cells from oxidative stress $[9,10]$. Studies also showed the effectiveness of CoQ10 on glycemic control with reduced $\mathrm{HbA}_{1 \mathrm{c}}$ levels. It is said to have protective effect on $\beta$ cells by virtue of its antioxidant potential [11].

$\mathrm{N}$-acetylcysteine (NAC) is a cysteine and glutathio- ne precursor in mammals that serves as powerful antioxidant. It also shows protective action on the $\beta$-cells. NAC administration decreases hyperglycemia with the improvement in the glucose intolerance associated with the glucose-induced insulin secretion [12]. NAC is also found beneficial in attenuating renal injury by protecting renal structure by decreasing the apoptosis due to ROS. NAC is proved advantageous in patients with DN as it acts through dual way by reducing both hyperglycemia and renal oxidative stress [12-14].

Therefore, an effort was taken to study the effect of antioxidants like CoQ10 and NAC in renoprotection against experimentally induced DN. Hence, the present study was aimed to investigate the renoprotective effect of CoQ10 alone and in combination with NAC in streptozotocin (STZ) induced DN.

\section{Materials and Methods}

\section{Drugs and chemicals}

Coenzyme Q10, as a gift sample was obtained from Zydus Cadila, Ahmedabad, India. $\mathrm{N}$-acetylcysteine was procured from Loba Chemie (Mumbai, India). STZ was purchased from Sigma (USA). Spectrophotometric kits for assessment of superoxide dismutase (SOD), malondialdehyde (MDA), reduced glutathione (GSH), catalase (CAT), myeloperoxidase (MPO) and nitric oxide (NO) were purchased from Elabscience Biotechnology Inc., USA. All other Biochemical kits for estimation of total protein, albumin, creatinine, urea, BUN and uric acid, used in the study were procured from SPAN Diagnostics, India. All other chemicals and reagents used in the study were of analytical grade.

\section{Experimental animals}

Study was conducted using adult male Sprague-Dawley rats (8-weeks-old, weighing 220-250 g). Animals were procured from the National Institute of Bioscience, Pune and housed under standard conditions in polypropylene cages. Rats were housed in a controlled environment of temperature $\left(18-22^{\circ} \mathrm{C}\right)$ and light (12-hr light/dark cycle, lights on 07:00-19:00). All animals were given ad libitum access to standard food and water and were acclimated for 1 week prior to the beginning of the study. All the procedures which applied to rats in this work were performed in accordance with ethical guidelines on the care and use of animals issued by Committee for the Purpose of Control and Supervision of Experiments on Animals (CPCSEA). The protocol of the study was approved by Institutional Animal Ethics Committee (IAEC).

\section{Induction of diabetic nephropathy}

T1DM was induced in overnight fasted 40 adult male Sprague-Dawley rats (220-250 g). The rats received 55 $\mathrm{mg} / \mathrm{kg}$ streptozotocin as a single intraperitoneal (i.p.) injection. STZ was administered immediately after dissolving it in freshly prepared cold citrate buffer, $(\mathrm{pH}$ 
4.5). Hyperglycemia was assessed at $72 \mathrm{~h}$ and then on day 7 post-STZ injection by determination of blood glucose using glucometer (AlereG1, Korea) in samples gathered from the end part of tails. Animals with blood glucose level greater than $280 \mathrm{mg} / \mathrm{dL}$ were considered as diabetic and included in the DN studies.

\section{Experimental design}

Rats were randomly divided into five groups each consisting six animals.

Group I: Normal control rats.

Group II: Diabetic control rats.

Group III: Diabetic rats treated with $10 \mathrm{mg} / \mathrm{kg}$ CoQ10 suspended in $1 \%$ aqueous solution of Tween 80 , p.o. $[11,15]$.

Group IV: Diabetic rats treated with NAC dissolved in distilled water (300 mg/kg, p.o) $[16,17]$.

Group V: Diabetic rats treated with a combination of CoQ10 (10 mg/kg) and NAC (300 mg/kg).

Treatments of Diabetic animals were started 4 weeks after injection of STZ. All the treatments were given daily to the respective group of animals for 8 weeks.

At the end of the protocol (12 weeks), and Twenty-four hours after the last antioxidant dose, animals were placed individually in metabolic cages for 24-hour urine collection. The urine samples were centrifuged and stored in amber colored vials. After that, animals were anesthetized for blood collection via retro orbital plexus. Blood samples thus obtained were stored in commercially available tubes with or without ethylene diamine tetra-acetic acid (EDTA). Serum was separated using standard protocol. Both serum and urine samples utilized for biochemical estimations were stored at $-20^{\circ} \mathrm{C}$.

$\mathrm{HbA}_{1 \mathrm{C}}$ was estimated using whole blood. Total protein, albumin, creatinine, urea, and uric acid were estimated from serum and urine samples using standard diagnostic kits with the help of Prietest Touch Biochemistry Analyser, Robonik India Pvt Ltd. (Mumbai, India).

At the end, rats were sacrificed and the kidneys were excised. Right kidney was removed and prepared for histological sections stained with hematoxylin-eosin. Another kidney was used and processed for estimation of antioxidant enzymes.

\section{Estimation of biomarkers of oxidative stress}

Kidney was removed and kept on petriplate in icecold conditions. The tissues were chopped using surgical scalpel into fine slices in chilled $0.25 \mathrm{M}$ sucrose, quickly blotted on filter paper. They were minced and homogenized in either $10 \mathrm{mM}$ Tris-HCl buffer, $\mathrm{pH} 7.4$ or normal saline or phosphate buffered saline (PBS). A 10\% $\mathrm{w} / \mathrm{v}$ tissue homogenate was prepared as per the protocol supplied with respective antioxidant enzyme assay kit. Tissue homogenate was prepared with 25 strokes of tight Teflon pestle of glass homogenizer at a speed of $10,000 \times \mathrm{g}$ at $0{ }^{\circ} \mathrm{C}$ using the Remi cooling centrifuge. A clear supernatant thus obtained was used for measurements of SOD (E-BC-K022), CAT (E-BC-K031), GSH (E$B C-K 051), M D A(E-B C-K 025), M P O(E-B C-K 074)$ and NO (E-BC-K035).

\section{Histopathology}

Kidney tissues thus obtained from different treatment groups were dissected, washed immediately using saline. $10 \%$ phosphate buffered formalin was used to fix the tissue. Kidney specimens were embedded in paraffin before cut into $5 \mu \mathrm{m}$-thick sections. The cut sections were stained with hematoxylin and eosin (H\&E and examined under the light microscope (Motic DMWB-1 Professional B3 Series, China). The observations were recorded for the presence of histopathological changes in the kidney and photomicrographs were taken.

\section{Statistical analysis}

All the data are expressed as mean \pm SEM. Statistical significance was tested using one-way ANOVA followed by the Tukey's multiple comparisons test as appropriate using Prism, GraphPad version 5, GraphPad Software, Inc. The significance was set at $p<0.05$ for all tests.

\section{Results}

\section{Effects of CoQ10 and/or NAC treatment on body weight}

There were significant differences in the body weight when all groups were compared $(P<0.05)$ (Table 1$)$. In rats of control group, there is a significant $(P<0.05)$ increase in body weight at the twelfth week. On the other hand, diabetic rats showed a significant decrease $(P<$ 0.05 ) in body weight at the same period. The treatment with CoQ10 or NAC and CoQ10 + NAC showed reduced weight loss as compared with diabetic rats received STZ. Also there is a significance effect of combined treatment of CoQ10 and NAC in diabetic animals than rats treated with either CoQ10 or NAC alone (Table 1).

\section{Effects of CoQ10 and/or NAC treatment on kidney to body weight ratio}

STZ induced diabetic rats showed a significant $(\mathrm{P}<$ 0.05 ) increase in kidney to body weight ratio as compared to rats in control group. Administration of CoQ10 (10 mg/kg, p.o.), NAC (300 mg/kg, p.o) and the combination of CoQ10 and NAC after induction of DN to the diabetic rats for eight weeks prevented kidney hypertrophy significantly $(P<0.05)$ as indicated by increased kidney to body weight ratio (Table 1 ).

\section{Effect of administration of CoQ10, NAC alone or their combination on blood glucose level and $\mathrm{Hb} \mathrm{A}_{1 \mathrm{C}}$}

As shown in Table 2, after $72 \mathrm{~h}$ of STZ injection, significant rise in blood glucose level was observed in all 
the animals at week 0 when compared to the control animals. This hyperglycemia became persistent in diabetic rats till the end of $12^{\text {th }}$ Week compared to control rats. CoQ10 $(10 \mathrm{mg} / \mathrm{kg}$, p.o. $)$ treatment from $5^{\text {th }}$ to $12^{\text {th }}$ week showed significant reduction in blood glucose $(P<$ $0.05)$ in comparison to the diabetic animals. Also there was significant $(P<0.05)$ difference in blood glucose at week 0 and week 12 in animals receiving CoQ10 alone. NAC at dose of $300 \mathrm{mg} / \mathrm{kg}$, p.o. showed significant $(P<$ $0.05)$ restriction of hyperglycemia as compared to animals with diabetes but failed to reduce elevated blood glucose levels when compared to results obtained with animals from NAC treated group at week 0 (Table 2). Combination of CoQ10 and NAC showed significant $(\mathrm{P}<$ 0.05 ) attenuation of hyperglycemia as compared to all other diabetic groups with or without treatment included in the study. Also there was significant $(P<0.05)$ difference in blood glucose at week 0 and week 12 after treatment of diabetic animals with the combination of CoQ10 and NAC.
The levels of $\mathrm{HbA}_{1 \mathrm{C}}$ (Table 2) were significantly ( $\mathrm{P}$ $<0.05)$ increased in STZ treated rats, as compared to normal control rats. CoQ10 $(10 \mathrm{mg} / \mathrm{kg})$ treated group showed significant decrease in $\mathrm{HbA}_{1 \mathrm{C}}$ when compared to diabetic control rats. Also NAC when administered at dose of $300 \mathrm{mg} / \mathrm{kg}$ for 8 weeks significantly attenuated glycated hemoglobin concentration as compared to the STZ treated diabetic rats. Animals in the group receiving both CoQ10 and NAC showed significant reduction of $\mathrm{HbA}_{1 \mathrm{C}}$ levels in comparison to the diabetic rats receiving either CoQ10 or NAC alone (Table 2).

\section{Effect of Coenzyme Q10, NAC or Combination of on serum total protein, albumin, creatinine, and creatinine clearance}

8 weeks post STZ injection showed a marked reduction in renal function characterized by significant $(p$ $<0.005)$ decrease in serum parameters like total protein, albumin and creatinine clearance (Table 3). On the other hand the serum concentration of creatinine incre-

Table 1: Effect of coenzyme Q10, NAC or combination of both on body weight and kidney to body weight ratio (mg/g).

\begin{tabular}{|c|c|c|c|}
\hline \multirow[t]{2}{*}{ Groups } & \multicolumn{2}{|l|}{ Body weight } & \multirow[t]{2}{*}{ Kidney to Body Weight Ratio $(\mathrm{mg} / \mathrm{g})$} \\
\hline & Initial at Week 0 & Final at Week 12 & \\
\hline Control & $242.3 \pm 0.919$ & $351.7 \pm 2.093^{*}$ & $3.807 \pm 0.041$ \\
\hline STZ DN & $247.2 \pm 1.796$ & $210.7 \pm 2.906^{a^{*}}$ & $10.45 \pm 0.069^{a}$ \\
\hline CoQ10 (10 mg/kg) & $249.0 \pm 1.125$ & $231.8 \pm 0.749^{a b^{*}}$ & $6.800 \pm 0.131^{a b}$ \\
\hline NAC (300 mg/kg) & $249.0 \pm 1.751$ & $227.0 \pm 1.983^{a^{b^{*}}}$ & $7.098 \pm 0.202^{\mathrm{ab}}$ \\
\hline CoQ10 + NAC & $246.2 \pm 1.905$ & $251.8 \pm 1.642^{\mathrm{abcd}^{*}}$ & $6.025 \pm 0.174^{\mathrm{abcd}}$ \\
\hline
\end{tabular}

Values are expressed as mean $\pm \mathrm{SEM} ; n=6$; $\mathrm{a}: \mathrm{P}<0.001$ as compared to normal control group; $\mathrm{b}$ : $\mathrm{P}<0.001$ as compared to diabetic control group; c: $\mathrm{P}<0.001$ as compared to diabetic CoQ10 alone treated group; $\mathrm{d}$ : $\mathrm{P}<0.001$ as compared to diabetic NAC alone treated group; ${ }^{P}<0.05$ compared with the same group at 0 week.

Table 2: Effect of CoQ10, NAC or their combination on blood glucose level (mg/dL) and $\mathrm{Hb} \mathrm{A}_{1 \mathrm{C}}$.

\begin{tabular}{|c|c|c|c|}
\hline \multirow[t]{2}{*}{ Groups } & \multicolumn{2}{|c|}{ Blood Glucose (mg/dL) } & \multirow[t]{2}{*}{$\mathrm{Hb} \mathrm{A}_{1 \mathrm{c},} \%$} \\
\hline & Initial at Week 0 & Final at Week 12 & \\
\hline Control & $91.83 \pm 2.937$ & $91.0 \pm 3.13$ & $4.73 \pm 0.084$ \\
\hline STZ DN & $493.5 \pm 6.722^{\mathrm{a}}$ & $525.5 \pm 13.75^{a}$ & $12.73 \pm 0.434^{a}$ \\
\hline CoQ10 (10 mg/kg) & $489.0 \pm 12.25^{a}$ & $427.7 \pm 11.81^{\mathrm{ab}^{*}}$ & $8.35 \pm 0.345^{\mathrm{ab}}$ \\
\hline NAC (300 mg/kg) & $485.3 \pm 11.06^{a}$ & $446.5 \pm 12.92^{\mathrm{ab}}$ & $8.96 \pm 0.332^{\mathrm{ab}}$ \\
\hline CoQ10 + NAC & $483.2 \pm 14.30^{\mathrm{a}}$ & $379.3 \pm 10.44^{\mathrm{abcd}^{*}}$ & $6.95 \pm 0.266^{\mathrm{abcd}}$ \\
\hline
\end{tabular}

Values are expressed as mean $\pm \mathrm{SEM} ; n=6$; $\mathrm{a}: \mathrm{P}<0.001$ as compared to normal control group; $\mathrm{b}$ : $\mathrm{P}<0.001$ as compared to diabetic control group; c: $\mathrm{P}<0.001$ as compared to diabetic CoQ10 alone treated group; $\mathrm{d}$ : $\mathrm{P}<0.001$ as compared to diabetic NAC alone treated group; ${ }^{*} \mathrm{P}<0.05$ compared with the same group at 0 week.

Table 3: Effect of CoQ10, NAC or their combination on serum total protein, albumin, creatinine and creatinine clearance.

\begin{tabular}{|l|l|l|l|l|}
\hline Groups & Total Protein $(\mathbf{g} / \mathbf{d L})$ & Albumin $(\mathbf{g} / \mathbf{d L})$ & Creatinine $(\mathbf{m g} / \mathbf{d L})$ & Creatinine clearance $(\mathbf{m L} / \mathbf{m i n})$ \\
\hline Control & $7.55 \pm 0.071$ & $3.65 \pm 0.042$ & $0.426 \pm 0.032$ & $1.134 \pm 0.088$ \\
\hline STZ DN & $4.83 \pm 0.152^{\mathrm{a}}$ & $1.55 \pm 0.154^{\mathrm{a}}$ & $2.96 \pm 0.091^{\mathrm{a}}$ & $0.238 \pm 0.017^{\mathrm{ab}}$ \\
\hline CoQ10 $(10 \mathrm{mg} / \mathrm{kg})$ & $6.25 \pm 0.152^{\mathrm{ab}}$ & $2.46 \pm 0.055^{\mathrm{ab}}$ & $1.002 \pm 0.050^{\mathrm{ab}}$ & $0.615 \pm 0.038^{\mathrm{ab}}$ \\
\hline NAC $(300 \mathrm{mg} / \mathrm{kg})$ & $6.13 \pm 0.269^{\mathrm{ab}}$ & $2.61 \pm 0.094^{\mathrm{ab}}$ & $1.06 \pm 0.046^{\mathrm{ab}}$ & $0.564 \pm 0.052^{\mathrm{ab}}$ \\
\hline CoQ10 + NAC & $6.78 \pm 0.119^{\mathrm{abd}}$ & $2.96 \pm 0.071^{\mathrm{abc}}$ & $0.78 \pm 0.036^{\mathrm{abcd}}$ & $0.869 \pm 0.083^{\mathrm{abcd}}$ \\
\hline
\end{tabular}

Values are expressed as mean \pm SEM; $n=6$; a: $\mathrm{P}<0.001$ as compared to normal control group; $\mathrm{b}: \mathrm{P}<0.001$ as compared to diabetic control group; c: $\mathrm{P}<0.001$ as compared to diabetic $\mathrm{CoQ} 10$ alone treated group; $\mathrm{d}$ : $\mathrm{P}<0.001$ as compared to diabetic NAC alone treated group. 
ased as compared to normal control rats indicating the impairment of renal function due to single i.p injection of STZ. Treatment of diabetic rats with either CoQ10, NAC or combined administration of both resulted in restoration of total protein and albumin along with improved creatinine clearance (Table 3). Also the serum creatinine, levels were reduced significantly as compared to diabetic control rats.

The combination of CoQ10 and NAC showed more beneficial effect in reducing serum creatinine concentrations than that of diabetic animals receiving CoQ10 or NAC alone.

\section{Effect of coenzyme Q10, NAC or combination of both on serum urea, BUN and uric acid}

Animals treated with CoQ10 (10 mg/kg, p.o), NAC (300 mg/kg, p.o.) or CoQ10 + NAC showed a significant $(p<0.05)$ reduction in urea (Figure $1 A$ ) and BUN (Figure 1B) levels as compared to diabetic control rats. Treatment with CoQ10, NAC or their combination resulted in significant $(p<0.05)$ reduction in serum uric acid (Figure 1C) in comparison to diabetic control rats. It was also observed that, CoQ10 and NAC when given in combination to diabetic rats resulted in more beneficial effect in reducing serum urea, BUN and uric acid levels than rats receiving CoQ10 or NAC alone.

\section{Effect of CoQ10, NAC or combination of both on urine volume, urinary protein and albumin excre- tion}

$24 \mathrm{~h}$ urine volume $(\mathrm{mL})$ of rats received STZ was significantly $(p<0.05)$ increased as compared to the normal control rats. Diabetic rats treated with CoQ10, NAC or their combination, a significant $(p<0.05)$ reduction in urine volume was observed as compared to diabetic control rats without any treatment (Figure 2A).

At $12^{\text {th }}$ week post STZ injection, a significant $(p<0.05)$ increase in urinary protein of diabetic rats was observed as compared to normal control rats. The treatment of diabetic animals with CoQ10, NAC or their combination resulted in a significant $(p<0.05)$ reduction in urinary protein when compared to diabetic control rats (Figure 2B).

Urinary albumin excretion rate (UAER) of diabetic rats was significantly increased due to renal impairment in comparison to the control rats at $12^{\text {th }}$ week. The treatment of diabetic animals after fourth week to twelfth
A

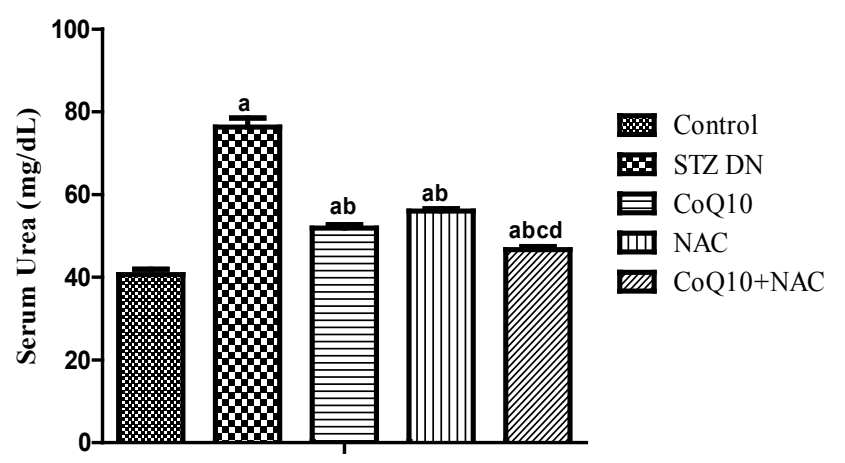

Groups
B

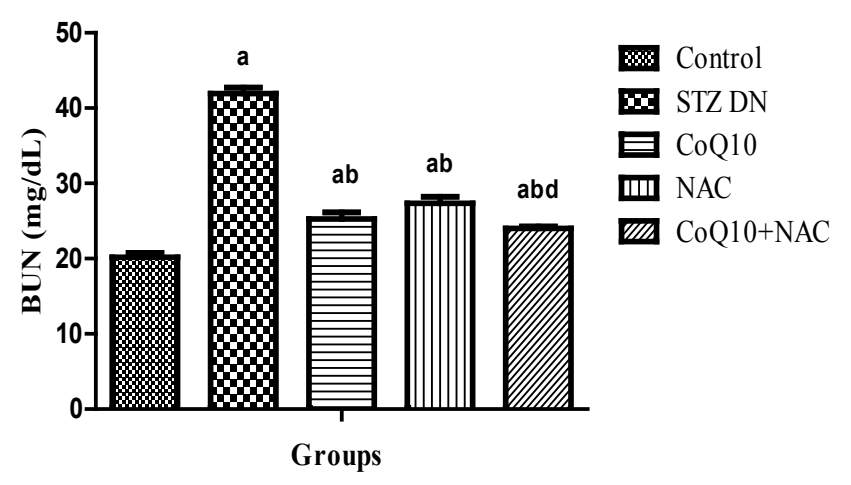

C

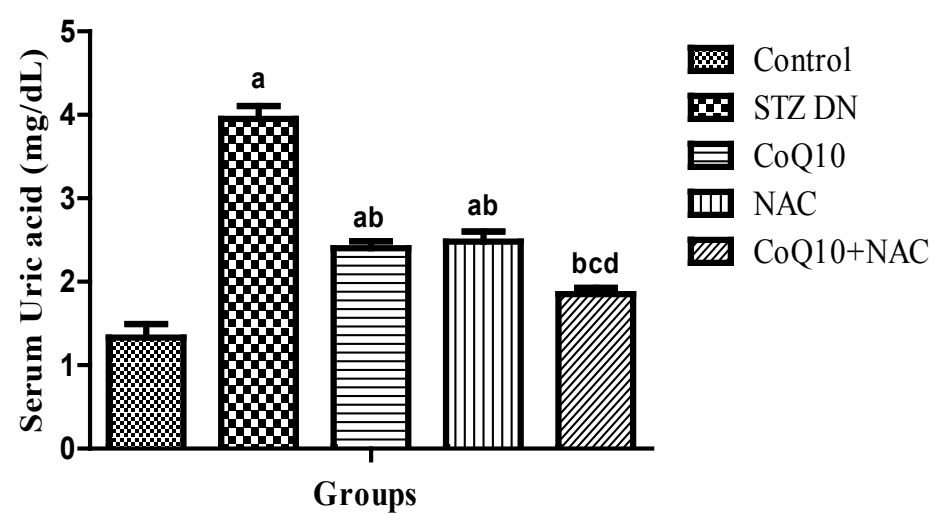

Figure 1: Effect of Coenzyme Q10, NAC or Combination of both on A) Serum urea, B) BUN and C) Uric acid.

Values are expressed as mean $\pm \mathrm{SEM} ; n=6$; $\mathrm{a}$ : $\mathrm{P}<0.001$ as compared to normal control group; $\mathrm{b}$ : $\mathrm{P}<0.001$ as compared to diabetic control group; c: $\mathrm{P}<0.001$ as compared to diabetic CoQ10 alone treated group; $\mathrm{d}$ : $\mathrm{P}<0.001$ as compared to diabetic NAC alone treated group. 
A

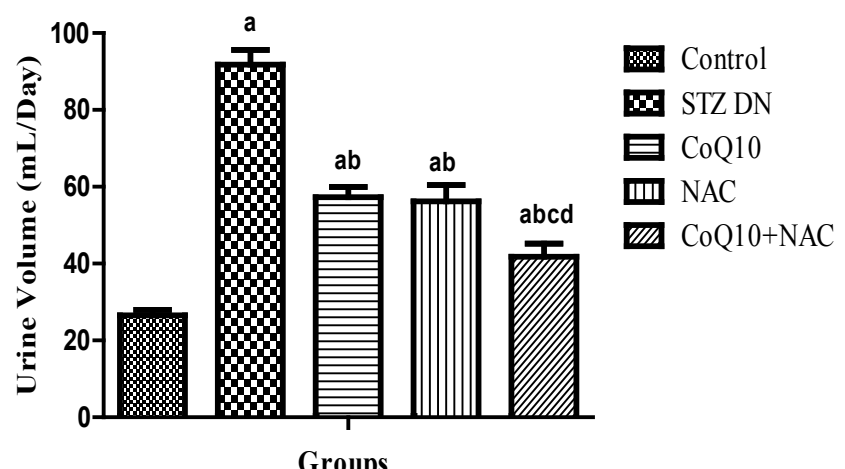

C

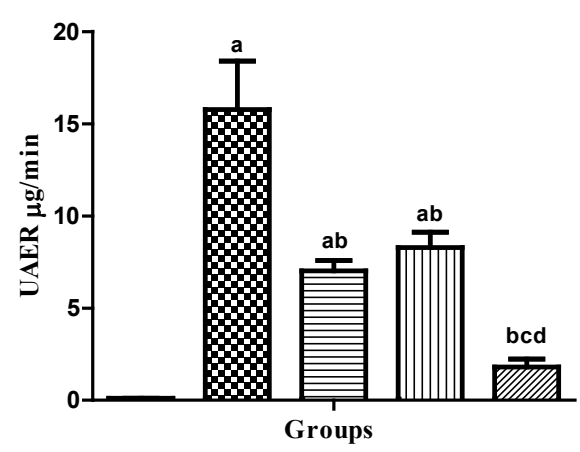

B

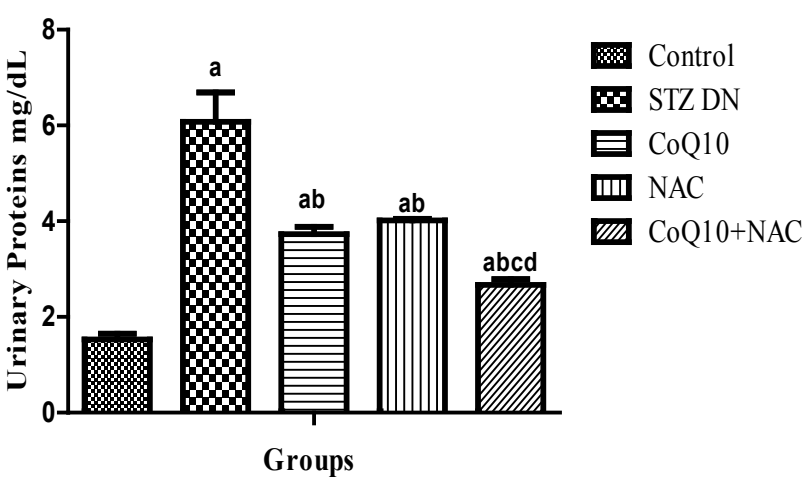

Figure 2: Effect of CoQ10, NAC or combination of both on A) Urine volume ( $\mathrm{mL} /$ Day), B) Urinary proteins $(\mathrm{mg} / \mathrm{dL})$ and $\mathrm{C})$ UAER ( $\mu \mathrm{g} / \mathrm{min})$.

Values are expressed as mean \pm SEM; $n=6$; $a$ : $P<0.001$ as compared to normal control group; $b$ : $P<0.001$ as compared to diabetic control group; c: $\mathrm{P}<0.001$ as compared to diabetic CoQ10 alone treated group; $\mathrm{d}$ : $\mathrm{P}<0.001$ as compared to diabetic NAC alone treated group.

week with CoQ10, NAC or their combination resulted in a significant $(p<0.05)$ reduction in UAER when compared to diabetic control rats (Figure $2 \mathrm{C}$ ).

It was also observed that, CoQ10 and NAC when given in combination to diabetic rats resulted in more beneficial effect as indicated by marked reduction UAER $\mu \mathrm{g} / \mathrm{min}$ (Figure $2 \mathrm{C}$ ).

\section{Effect of CoQ10, NAC or CoQ10 + NAC on renal oxi- dative stress and anti-oxidant markers}

The marker of lipid peroxidation, malondialdehyde (MDA) was a significantly $(p<0.05)$ increased in diabetic rats received injection of STZ. Diabetic animals receiving antioxidants CoQ10 (10 mg/kg, p.o.) and NAC (300 mg/ $\mathrm{kg}$, p.o.) showed reduced MDA levels significantly than untreated diabetic rats. The rats in the group treated with combination of CoQ10 and NAC for 8 weeks after induction of DN showed more beneficial effects in reducing levels of MDA in renal tissue (Figure 3A).

It was also evident that, the concentration of SOD (U/ $\mathrm{mg}$ Protein) and CAT (U/mg Protein) in renal homogenate of diabetic rats was significantly decreased indicating elevated oxidative stress in the animals of this group. The treatment with CoQ10, NAC or their combination (CoQ10 + NAC) ameliorated oxidative stress as indicated by significant elevation in the SOD and CAT concentra- tion in the renal tissue (Figure $3 \mathrm{~B}$ and Figure $3 \mathrm{C}$ ).

There was a significant reduction in reduced glutathione (GSH) in diabetic untreated group as compared to control group, administration of CoQ10, NAC or CoQ10 + NAC (10 and $300 \mathrm{mg} / \mathrm{kg}$ respectively) to the diabetic animals caused significant increase in the GSH level (Figure 3D).

\section{Effect of CoQ10, NAC or their combination of both on myeloperoxidase (MPO) activity and NO in re- nal tissue}

There was a significant increase in the renal MPO (U/g tissue) concentration at twelfth week in diabetic rats received STZ. 8 week treatment of diabetic rats with CoQ10, NAC alone or with their combination (CoQ10 + NAC) reduced the MPO activity as compared to the diabetic untreated rats. More advantageous observations were recorded in renal MPO levels when CoQ10 and NAC were given concomitantly to the diabetic rats than that of CoQ10 or NAC administered alone (Figure 3E).

Nitrite content was significantly $(p<0.05)$ increased in renal tissue of diabetic rats as compared to control group. Administration of CoQ10 or NAC alone to diabetic animals showed significant $(p<0.05)$ decrease in renal nitrite content as compared to diabetic untreated group. Group receiving CoQ10 + NAC showed more be- 
A

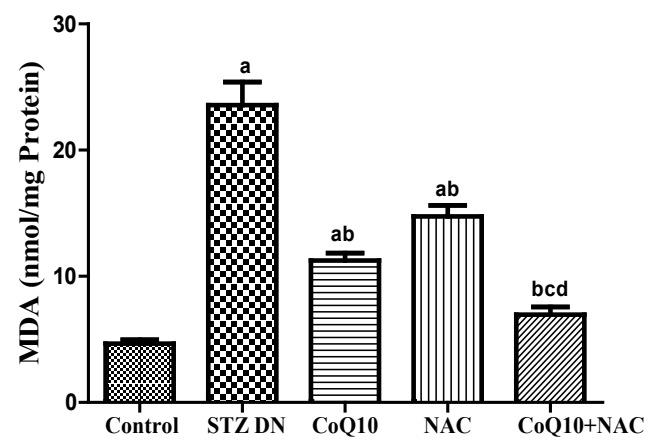

C

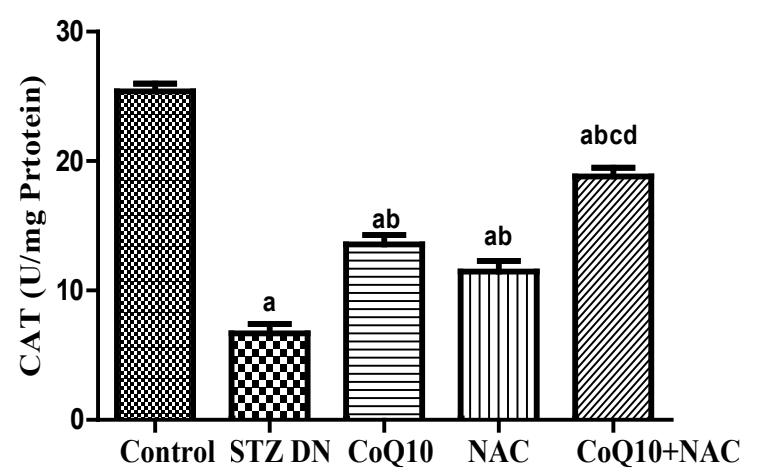

$\mathbf{E}$

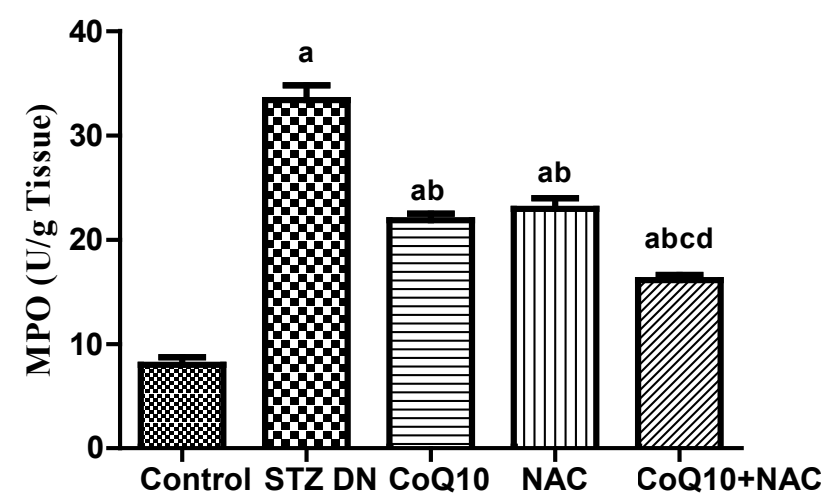

B

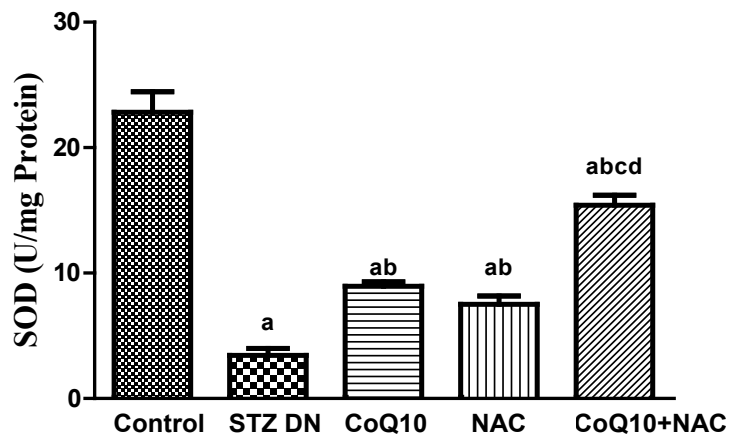

D

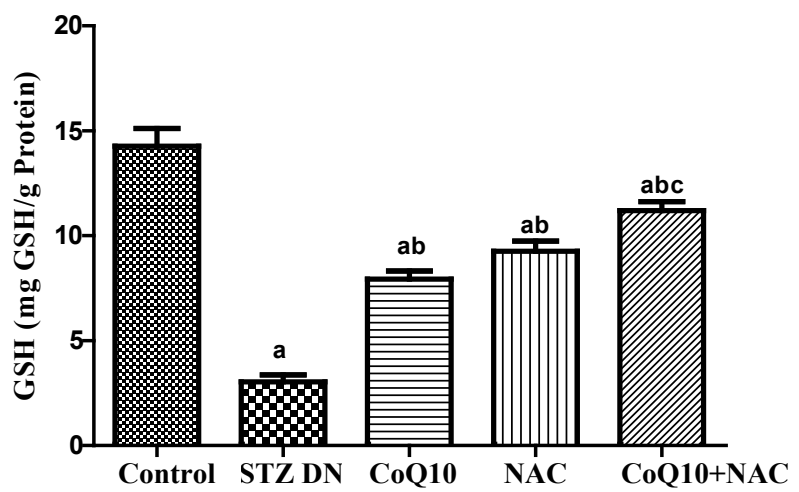

F

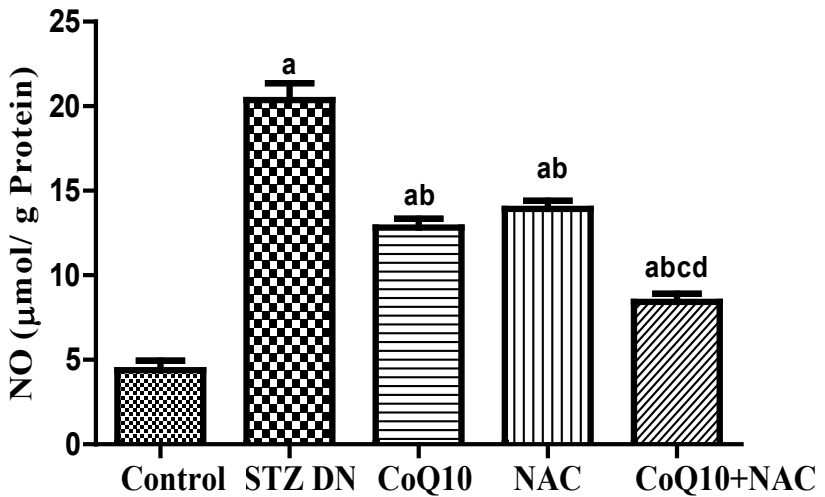

Figure 3: Effect of CoQ10, NAC or combination of both on renal oxidative stress and anti-oxidant markers in renal homogenate A) MDA, B) SOD, C) CAT, D) GSH, E) MPO and F) NO.

Values are expressed as mean $\pm \mathrm{SEM} ; n=6$; $\mathrm{a}$ : $\mathrm{P}<0.001$ as compared to normal control group; $\mathrm{b}$ : $\mathrm{P}<0.001$ as compared to diabetic control group; c: $\mathrm{P}<0.001$ as compared to diabetic CoQ10 alone treated group; $\mathrm{d}$ : $\mathrm{P}<0.001$ as compared to diabetic NAC alone treated group.

neficial effects on nitrite content in comparison to the diabetic rats treated with CoQ10 or NAC alone (Figure 3F).

\section{Effect of Co10, NAC or CoQ10 + NAC on histopa- thological changes of the kidney}

Renal tissues from all the five groups included in the study were stained by hematoxylin and eiosin. Light microscopic examination revealed normal histopathological appearance in the kidney specimen from control group in terms of glomerular basement membrane thickness and tubular degeneration. On the contrary,
STZ treated diabetic kidney showed glomerulosclerosis, thickened glomerular basement membrane and tubular degeneration and vacuolization. Renal tissue section of diabetic rats treated with CoQ10 or NAC showed improved histological feature with moderate alterations in glomerular and tubular appearance (Figure 4).

Interestingly, the treatment of diabetic rats with combination of CoQ10 and NAC showed renal histological appearance with very mild changes in morphology of glomerular basement membrane and tubular swelling (Figure 4). 

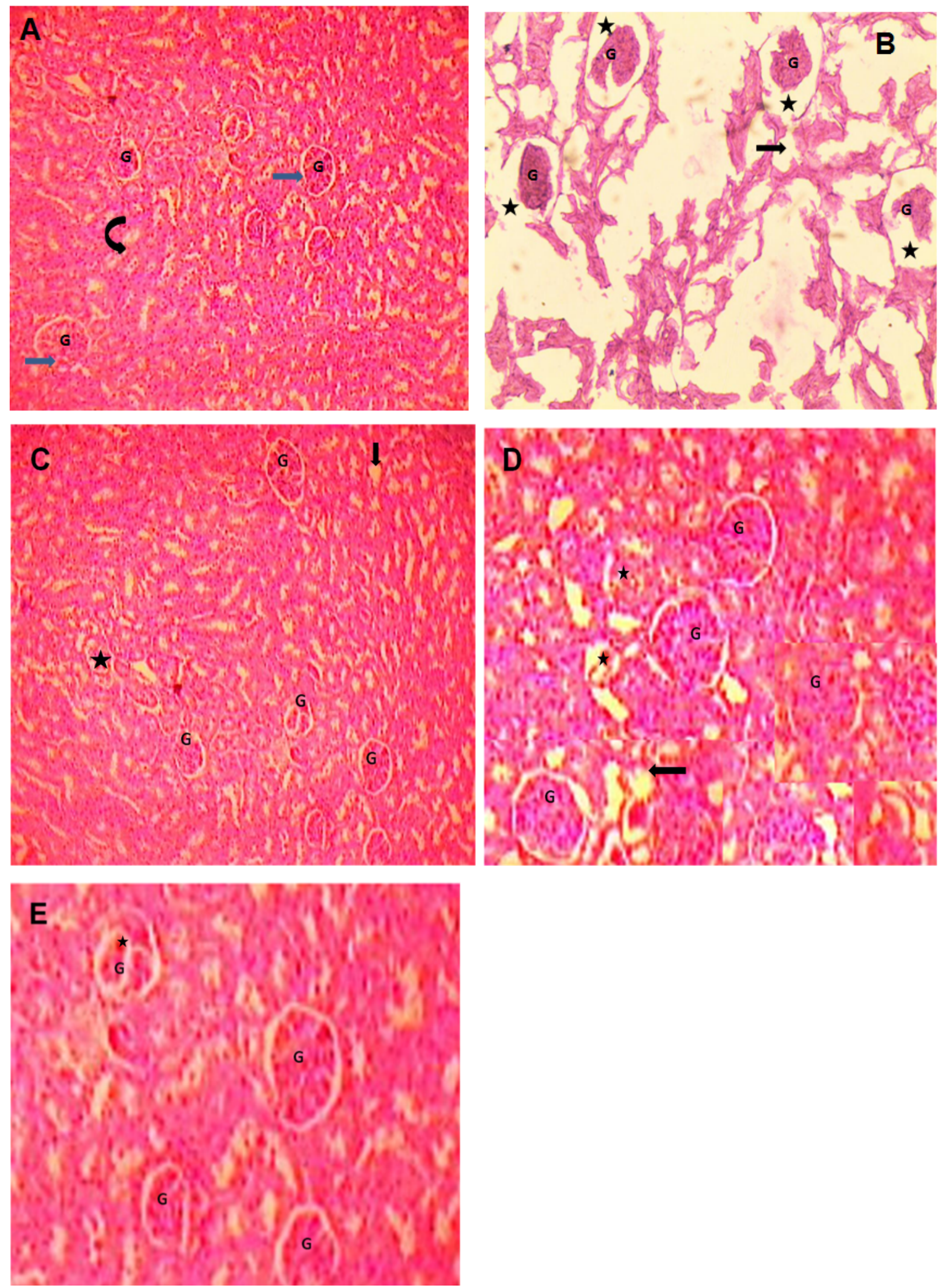

Figure 4: Photomicrographs of renal tissues from rats (Hematoxylin and Eosin staining) A) Control group, B) Diabetic group, C) Coenzyme Q10, D) NAC and E) CoQ10 + NAC

A) Section of the kidney showing the normal appearance of the glomerulus ( $G$, blue arrow) and renal tubules (curved arrow) in control rats; B) Representative sections of the diabetic rat kidney with most of the glomeruli (G) degenerated with wide urinary space (black star) indiacating increased glomerular basement membrane thickness. Tubular epithelial cells show vacuolar degeneration (black arrow) and sings of focal necrosis; C) Renal histology of STZ diabetic rat treated with CoQ10 with fewer glomeruli with degeneration (black star), vacuolar degeneration in some tubular epithelial cells (Black arrow); D) Kidney section from diabetic rat treated with NAC with some glomerular degeneration (black star), vacuolar degeneration in some tubular epithelial cells (Black arrow); E) Renal histology of diabetic rat treated with combination of CoQ10 + NAC showing normal appearance of the glomerulus $(G)$ with fewer glomeruli with lobulation. Tubular epithelial cells appear normal with less degree of vacuolization. 


\section{Discussion}

Diabetic nephropathy (DN) is rapidly becoming the major cause of end-stage renal disease (ESRD) worldwide. It is considered that the principal player of all diabetic complications including DN is persistent hyperglycemia. Also, this chronically elevated blood glucose level is the single most important factor in the generation of early and sustained oxidative stress (OS) during DM. OS along with chronic hyperglycemia plays an important role in the pathogenesis of glomerular and tubular functional and structural abnormalities, even before the onset of microalbuminuria. During DM, chronic hyperglycemia is shown to induce OS through diverse mechanisms including glucose auto-oxidation, protein glycation through non-enzymatic means, polyol pathway activation and acceleration, and reduced antioxidant defense system [8]. It is also evident that elevated blood glucose lead to the formation of AGEs and the mitochondrial production of free radicals and consequently, to cell death and renal dysfunction. Thus, oxidative stress plays a crucial role in the development of DN.

Therefore, one of the different approaches to overcome DM and its complications is through use of variety of antioxidant molecules which are found to be effective in alleviating diabetic complications including nephropathy. These agents not only capable of scavenging the free radicals activity, some antioxidants are shown of modulate various signaling pathways and restoring the normal renal function. Also T1DM, which is associated with autoimmunity, is showed to generate OS by many different mechanisms. Currently, many antioxidants with an ability to overcome autoimmune disease, inhibit inflammatory process and scavenge free radicals are available. These agents are proved advantageous in diabetes induced complications especially DN. Antioxidants such as vitamin $\mathrm{E}$, vitamin $\mathrm{C}$, coenzyme Q10, N-acetylcysteine, alpha-lipoic acid, taurine and many others protect the cells against oxidative stress $[18,19]$. Therefore, the use of antioxidants as a complementary therapy is useful in oxidative stress-related diseases including DN.

The present investigation demonstrates the renoprotective potential of antioxidants coenzyme Q10 and $\mathrm{N}$-acetylcysteine when given alone and in combination against streptozotocin induced diabetic nephropathy in rats.

In the present study, renal function of diabetic rats was significantly impaired as indicated by renal function tests than control rats. Renal damage caused due to STZ was identified by means of increased MDA, depleted SOD and CAT activities and reduced GSH. MPO activity and nitrite content in rats with DN increased significantly. Treatment with CoQ10 or NAC and their combined treatment improved STZ induced renal damage as reflected by reduced oxidative stress.

Present study showed significant reduction in body weight of diabetic rats. This decrease may be related to degradation of structural proteins contributing to body weight. In STZ induced experimental DM, loss of body weight is associated with excessive breakdown of tissue proteins and increased muscle degeneration. Also, insulin absence leads to reduced protein synthesis with low serum total protein levels in diabetic animals [20]. Treatment of diabetic animals with CoQ10, NAC or their combination improved the body weight in diabetic rats in comparison to the rats received STZ.

The kidney weight/body weight ratio was significantly increased in the diabetic rats in comparison to control group. It is previously shown that renal structural changes such as glomerular basement membrane thickening and mesangial matrix expansion due to persistent hyperglycemia probably contributed to the increased kidney weights. It has been described that kidney hypertrophy leads to increased kidney weight in DM due to glucose over-utilization, glycogen accumulation, lipogenesis and protein synthesis in the renal tissue $[21,22]$. Treatment of diabetic rats with CoQ10, NAC or their combination successfully prevented the kidney enlargement.

Hyperglycemia due to diabetes is at center in the development and progression of nephropatia deabetica or Kimmelstiel-Wilson syndrome i.e. DN [23]. In this study, diabetic rats showed increased blood glucose level throughout the study period i.e. from week 0 to week 12. Also, $\mathrm{HbA}_{1 \mathrm{c}}$ was elevated in all diabetic animals. Diabetic rats receiving CoQ10, NAC or their combination from $5^{\text {th }}$ to $12^{\text {th }}$ week showed pronounced reduction in elevated blood glucose and glycated hemoglobin.

It is reported that, a decrease in total protein and albumin due to their excessive excretion via urine, play a role in the pathogenesis of DN [24]. Administration of the CoQ10, NAC or their combination resulted in restoration of total protein and albumin by preventing their renal loss. The combination of CoQ10 and NAC showed more beneficial effects in preventing loss of serum total proteins and albumin in comparison to diabetic animals receiving either CoQ10 or NAC alone.

Increased serum creatinine ( $\mathrm{SCr}$ ) concentration and creatinine clearance $(\mathrm{Ccr})$ are the most common characteristics in the development of DN [25]. The results of the study presented here demonstrate that rats with DN showed significant increases in the $\mathrm{SCr}$, and reduced $\mathrm{CCr}$ as compared with control rats, indicative of declined renal function. However, treatment with CoQ10, NAC or their combination positively affected these parameters, especially in the group received the combined treatment with CoQ10 and NAC.

Serum urea, BUN and uric acid levels are considered as markers of renal function test significantly increased in diabetic animals in comparison with the control group. This increase can be suggested to be due to deteriorated excretory and regulatory renal function to 
maintain a constant homeostasis of these parameters. 8 week treatment with CoQ10 and NAC or their concomitant administration significantly decreased serum urea, BUN and uric acid level in diabetic animals in comparison with the untreated diabetic animals. Several studies showed that natural antioxidants such as selenium, vitamin E, vitamin C [26], alpha-lipoic acid [18], coenzyme $\mathrm{Q} 10$ and rosmarinic acid decrease serum urea and creatinine in diabetic rats [27].

Along with chronic hyperglycaemia, polyuria is another feature of DM due to osmotic diuresis. The increased urine volume (24 h) observed in this study was in accordance with osmotic diuresis associated with glucosuria. Also, there is significant increase in the urinary total proteins and urinary albumin excretion rate (UAER) indicating severe renal impairment. Reduced serum albumin increased UAER in diabetic complications have been associated with faster kidney disease progression. Previous studies showed that, DM induced renal dysfunction lead to gradual loss of serum albumin as a result of loss of negatively charged glycosaminoglycans in the cellular basement membrane and the subsequent enlargement of basement membrane pore size, thus leading to albuminuria $[28,29]$. Combined treatment with CoQ10 and NAC showed more beneficial effects in reducing urinary loss of plasma proteins along with reduction in UAER in diabetic animals.

Recent preclinical and clinical studies have shown that CoQ10 has a beneficial effect in decreasing the elevated $\mathrm{HbA}_{1 \mathrm{C}^{\prime}}$ urea, and creatinine in diabetes [27,30,31]. Also, studies with NAC revealed its potential in attenuating renal injury induced by DM along with reduction in urinary protein and urinary thiobarbituric acid reactive substances $[14,16]$.

OS is considered as an important factor in development of DN and underlying kidney damage. In Diabetic state, OS is induced due to disturbance in the normal antioxidant defense and increased free-radical formation which further initiates multiple downstream signaling mechanisms that interfere with normal renal structural and functional features. It has been showed by various independent research groups that there is improvement in the renal physiology in DN after antioxidants treatment that act directly against oxidative tissue damage. Moreover, antioxidants administered exogenously are capable of providing protection against progression of DN. Antioxidant do this by blocking the formation of excessive reactive oxygen species (ROS) effectively and scavenging the preformed intracellular ROS [32].

Several reports showed that natural antioxidants such as vitamin E, vitamin C, coenzyme Q10, lipoic acid, aminoguanidine, turine, $\mathrm{N}$-acetylcysteine and antioxidative enzymes such as SOD, CAT, and GSH have pro- tective effects against OS induced renal damage in DM [27,33].

Results in the present study showed an increase in the OS after STZ induced DN as indicated by elevated MDA and reduced SOD, CAT, and GSH levels. There were significant reduction in lipid peroxidation and an increase in SOD, CAT, and GSH levels after the treatment with CoQ10, NAC or combination of both. Combined effect of both the antioxidants (CoQ10 + NAC) has shown more beneficial effect in altering MDA, SOD, CAT and GSH levels than when administered alone.

There exists the relationship between myeloperoxidase (MPO) levels and DM with a positive correlation between elevated MPO levels and development of DN [34]. In present study, there was a significant decrease in renal MPO level in CoQ10 and NAC alone treated rats. Moreover, CoQ10 in combination with NAC showed more significant decrease in renal MPO levels than the groups received treatment either with CoQ10 or NAC alone. On the contrary, a better renoprotective effect showed by significant reduction of MPO levels in renal tissue when CoQ10 and NAC combination therapy is given to rats with $\mathrm{DN}$.

Modulation of renal structure and function in diabetes is associated with abnormal nitric oxide (NO) synthesis. Accumulating research showed that levels of nitrite, an oxidized end product of NO, which might be attributed to the formation of peroxynitrite by reaction of NO with generated superoxide radicals $[35,36]$. In the present study, diabetic rats showed a significant increase in tissue nitrite content as compared to normal control rats. Whereas renal nitrite levels found to be restored in animals treated with CoQ10 or NAC or CoQ10 + NAC.

\section{Conclusion}

The finding of the current study showed that the treatment with a natural antioxidant coenzyme Q10 or $\mathrm{N}$-acetylcysteine possess significant renoprotective effect against streptozotocin induced DN. Conversely, combined administration of CoQ10 and NAC showed a more beneficial and renoprotective effect than coenzyme Q10 or NAC alone which may be attributed to improved renal function along with reduction in oxidative stress as indicated by amelioration of lipid peroxidation and suppressed MPO activity in addition to nitrite content in renal tissue. Finally, it was concluded that combined administration of coenzyme Q10 with $\mathrm{N}$ - acetylcysteine might attenuate or delay the progression of DN.

\section{Acknowledgements}

Authors are thankful to Zydus Cadila, Ahmedabad, India. For providing coenzyme Q 10 as gift sample.

\section{Conflicts of Interest}

Authors declare that no conflict of interest. 


\section{References}

1. American Diabetes Association (2008) Diagnosis and classification of diabetes mellitus. Diabetes Care 31: S55-S60.

2. Ramachandran A, Snehalatha $C$, Shetty AS, Nanditha A (2012) Trends in prevalence of diabetes in Asian countries. World J Diabetes 3: 110-117.

3. Pal PB, Sinha K, Sil PC (2014) Mangiferin attenuates diabetic nephropathy by inhibiting oxidative stress mediated signaling cascade, TNFa related and mitochondrial dependent apoptotic pathways in streptozotocin-induced diabetic rats. PLoS One 9: e107220.

4. Mogensen CE, Christensen CK, Vittinghus E (1983) The stages in diabetic renal disease. With emphasis on the stage of incipient diabetic nephropathy. Diabetes 32: 64-78.

5. Vora JP, Ibrahim HAA (2003) Clinical manifestations and natural history of diabetic nephropathy. In: Johnson RJ, Feehally J, Comprehensive clinical nephrology. ( $2^{\text {nd }}$ edn), Mosby, Edinburgh, 425-437.

6. Schena FP, Gesualdo L (2005) Pathogenetic mechanisms of diabetic nephropathy. J Am Soc Nephrol 16: S30-S33.

7. Vasavada N, Agarwal R (2005) Role of oxidative stress in diabetic nephropathy. Adv Chronic Kidney Dis 12: 146-154.

8. Mahajan MS, Gulecha VS, Upaganlawar AB, Upasani CD (2019) Antioxidant defense in diabetic nephropathy. In: Uddin S, Upaganlawar AB, Oxidative stress and antioxidant defense: Biomedical value in health and diseases. $\left(1^{\text {st }} e d n\right)$, Nova, New York.

9. Hodgson JM, Watts GF, Playford DA, Burke V, Croft KD (2002) Coenzyme Q10 improves blood pressure and glycaemic control: A controlled trial in subjects with type 2 diabetes. Eur J Clin Nutr 56: 1137-1142.

10. Rosenfeldt FL, Haas SJ, Krum H, Hadj A, Ng K, et al. (2007) Coenzyme Q10 in the treatment of hypertension: A meta-analysis of the clinical trials. J Hum Hypertens 21: 297-306.

11. Maheshwari RA, Balaraman R, Sen AK, Seth AK (2014) Effect of coenzyme Q10 alone and its combination with metformin on streptozotocin-nicotinamide-induced diabetic nephropathy in rats. Indian J Pharmacol 46: 627-632.

12. Haber CA, Lam TK, Yu Z, Gupta N, Goh T, et al. (2003) $\mathrm{N}$-acetylcysteine and taurine prevent hyperglycemia-induced insulin resistance in vivo: Possible role of oxidative stress. Am J Physiol Endocrinol Metab 285: E744-E753.

13. Ahmad A, Mondello S, Di Paola R, Mazzon E, Esposito E, et al. (2012) Protective effect of apocynin, a NADPH-oxidase inhibitor, against contrast-induced nephropathy in the diabetic rats: A comparison with n-acetylcysteine. Eur J Pharmacol 674: 397-406.

14. Shimizu MH, Coimbra TM, de Araujo M, Menezes LF, Seguro AC (2005) N-acetylcysteine attenuates the progression of chronic renal failure. Kidney Int 68: 2208-2217.

15. Garjani A, Andalib S, Biabani S, Soraya H, Doustar Y, et al (2011) Combined atorvastatin and coenzyme Q10 improve the left ventricular function in isoproterenol-induced heart failure in rat. Eur J Pharmacol 666: 135-141.

16. Lee ES, Kim HM, Kang JS, Lee EY, Yadav D, et al. (2016) Oleanolic acid and $\mathrm{N}$-acetylcysteine ameliorate diabetic nephropathy through reduction of oxidative stress and endoplasmic reticulum stress in a type 2 diabetic rat model. Nephrol Dial Transplant 31: 391-400.

17. Odetti P, Pesce C, Traverso N, Menini S, Maineri EP, et al. (2003) Comparative trial of $\mathrm{N}$-acetyl-cysteine, taurine, and oxerutin on skin and kidney damage in long-term experimental diabetes. Diabetes 52: 499-505.

18. Ahmadi A, Mazooji N, Roozbeh J, Mazloom Z, Hasanzade J (2013) Effect of alpha-lipoic acid and vitamin E supplementation on oxidative stress, inflammation, and malnutrition in hemodialysis patients. Iran J Kidney Dis 7: 461-467.

19. Jemai H, El Feki A, Sayadi S (2009) Antidiabetic and antioxidant effects of hydroxytyrosol and oleuropein from olive leaves in alloxan-diabetic rats. J Agric Food Chem 57: 8798-8804.

20. Rajkumar L, Srinivasan N, Balasubramanian K, Govindarajulu $P$ (1991) Increased degradation of dermal collagen in diabetic rats. Indian J Exp Bio 29: 1081-1083.

21. Teoh SL, Abdulatiff A, Das S (2010) Histological changes in the kidneys of experimental diabetic rats fed with Momordical charantia (bitter gourd) extract. Rom J Morphol Embryol 51: 91-95.

22. Mogensen CE (1999) Microalbuminuria, blood pressure and diabetic renal disease: Origin and development of ideas. Diabetologia 42: 263-285.

23. Roy S, Trudeau K, Roy S, Behl Y, Dhar S, et al. (2010) New insights into hyperglycemia-induced molecular changes in microvascular cells. J Dent Res 89: 116-127.

24. Sun L, Halaihel N, Zhang W, Rogers T, Levi M (2002) Role of sterol regulatory element-binding protein 1 in regulation of renal lipid metabolism and glomerulosclerosis in diabetes mellitus. J Biol Chem 277: 18919-18927.

25. Brenner BM, Meyer TW, Hostetter TH (1982) Dietary protein intake and the progressive nature of kidney disease: The role of hemodynamically mediated glomerular injury in the pathogenesis of progressive glomerular sclerosis in aging, renal ablation, and intrinsic renal disease. $\mathrm{N}$ Engl $\mathrm{J}$ Med 307: 652-659.

26. Karabulut-Bulan O, Bolkent S, Yanardag R, Bilgin-Sokmen $B$ (2008) The role of vitamin C, vitamin $E$, and selenium on cadmium-induced renal toxicity of rats. Drug Chem Toxicol 31: 413-426.

27. Ahmadvand H (2012) Effects of coenzyme Q10 on hemoglobin A1C, serum urea and creatinine in alloxan-induced Type 1 diabetic rats. Iran J Pharmacol Ther 11: 64-67.

28. Mora-Fernández $C$, Domínguez-Pimentel $\mathrm{V}$, de Fuentes MM, Górriz JL, Martínez-Castelao A, et al. (2014) Diabetic kidney disease: From physiology to therapeutics. J Physiol 592: 3997-4012.

29. Haraldsson B, Sörensson J (2004) Why do we not all have proteinuria? An update of our current understanding of the glomerular barrier. News Physiol Sci 19: 7-10.

30. Maheshwari $R$, Balaraman $R$, Sen AK, Shukla D, Seth A (2016) Effect of concomitant administration of coenzyme Q10 with sitagliptin on experimentally induced diabetic nephropathy in rats. Ren Fail 39: 130-139.

31. Zhang X, Shi Z, Liu Q, Quan H, Cheng X (2019) Effects of coenzyme Q10 intervention on diabetic kidney disease: $A$ systematic review and meta-analysis. Medicine 98: e15850.

32. Agrawal N, Sadhukhan P, Saha S, Sil PC (2015) Therapeutic insights against oxidative stress induced diabetic nephropathy: A review. J Autoimmun Disod 1: 1.

33. Cornejo-Garcia JA, Mayorga C, Torres MJ, Fernandez TD, R-Pena R, et al. (2006) Anti-oxidant enzyme activities and expression and oxidative damage in patients with non-immediate reactions to drugs. Clin Exp Immunol 145: 287 295. 
34. Rovira-Llopis S, Rocha M, Victor M, Falcon R, de Pablo C, et al. (2013) Is myeloperoxidase a key component in the ROS-induced vascular damage related to nephropathy in type 2 diabetes? Antioxid Redox Signal 19: 1452-1458.

35. Prabhakar SS (2004) Role of nitric oxide in diabetic nephropathy. Semin Nephrol 24: 333-344.
36. Stamler JS, Singel DJ, Loscalzo J (1992) Biochemistry of nitric oxide and its redox-activated forms. Science 258: 1898-1902. 